\section{Ultrasound guided foam sclerotherapy of recurrent varices of the great and small saphenous vein: 5 -year follow up}

\author{
Patrizia Pavei, Maurizio Ferrini, \\ Giorgio Spreafico, Attilio Nosadini, \\ Andrea Piccioli, Enzo Giraldi, \\ Ugo Baccaglini \\ Centro Regionale Specializzato \\ Multidisciplinare per la Day Surgery, AO \\ Padova, Italy
}

\section{Abstract}

Ultrasound guided foam sclerotherapy (UGFS) proved to be effective in recurrent varices. In this observational study from 2006 and 2012 we treated 142 neovascularization, 155 inguinal recurrence and 28 popliteal recurrent varices. For neovascularization $0.3-0.5 \%$ polidocanol (POL) sclerosant foam (SF) was injected for vein having diameter $<3 \mathrm{~mm}$ and $0.5-1 \%$ POL or sodium tetradecylsulphate (STS) SF for higher vein diameters. The patients with residual sapheno-femoral or sapheno-popliteal junctions were treated with $1 \%$ STS SF for diameter up to $5 \mathrm{~mm}$, while for larger veins 3\% STS was used. From 1 to 3 sessions were necessary in both groups with 4 to $10 \mathrm{ml}$ injected per session. In the group of neovascularization the 3-5years follow up revealed good results in $90.8 \%$ of the cases. In the group of popliteal recurrences the 3-5 years follow up showed $60.7 \%$ of good results, while in the group of inguinal recurrences we observed $80 \%$ of good results at 3-5 years. We did not have major complication. As minor complications we had $0.2 \%$ of gastrocnemial vein thrombosis, $0.1 \%$ of minor neurological problems, $2.8 \%$ of superficial vein thrombosis, $3.9 \%$ of pigmentation and light to mild post-treatment pain in $16.5 \%$ of the cases. In conclusion our data show that UGFS is a well tolerate technique, preferred by previously operated patients, safe and easily repeatable with good medium-term results both in case of neovascularization and of recurrence from residual femoral or popliteal stump.

\section{Introduction}

Recurrent varices after surgery are a common, complex and costly problem. The percentage of recurrences varies from 20 to $80 \%$ according to the definition of recurrence and to the length of the follow up. ${ }^{1,2}$ Actually, data in literature often lack uniformity and are difficult to compare. . $^{2,3}$

The causes of recurrence are technical or tactical in approximately $29 \%$ of the cases, often because of failure to adequately strip a refluxing great saphenous vein or for the presence of a residual saphenous stump, while neovascularization, characterized by fragile, thin walled varices often not easy to treat with surgery and not eligible for endovascular treatments, is responsible for another $29 \%{ }^{4-6}$ On the other hand, $32 \%$ of recurrences are represented by varices in new sites, which can be related to the progression of the disease.,7,8 Another cause of recurrence is an incompetence of the deep venous system that can occur in post-thrombotic syndrome or for congenital reasons (aplasia or valvular agenesis). ${ }^{7}$ The aim of the treatment of recurrent varices is to control varicose disease and its symptoms and to prevent complications. Patients suffering from this condition often belong to class $\mathrm{C} 3$ to C6 of the Clinical-Etiology-Anatomy-Pathophysiology (CEAP) classification and they are often reluctant to undergo a new treatment. Ultrasound guided foam sclerotherapy (UGFS) proved to be effective in recurrent varices ${ }^{9,10}$ and in this observational study we report our five-year follow-up results of UGFS in the treatment of varicose recurrence after surgery.

\section{Materials and Methods}

The prospective cohort study included patients with recurrent varicose veins in the operated site (high ligation + saphenous stripping and phlebectomy). All patients underwent a color duplex ultrasound (CDU) investigation of the operated limb according to the standard;11 residual sapheno-femoral junction (SFJ) or sapheno-popliteal junction (SPJ) diameter was measured $2 \mathrm{~cm}$ below the saphenous junction, whereas veins due to neovascularization were measured at their proximal site near to the junction. After CDU examination patients were divided into 2 groups: i) recurrence related to groin/popliteal neovascularization; and ii) recurrence due to new varices emanating from an already operated junction (residual stump of SFJ or SPJ). Our study did not include residual veins or recurrences originating from other sites, such as from incompetent perforators. From 2006 to 2012 we treated 142 cases of recurrent varices related to inguinal neovascularization, 155 inguinal recurrences emanating from residual SFJ stump and 28 popliteal recurrences related to residual SPJ (Table 1).

UGFS was performed as single treatment
Correspondence: Patrizia Pavei, Centro Regionale Multidisciplinare Specializzato di Day Surgery, Azienda Ospedaliera di Padova, via Giustiniani 2, 35128 Padova, Italy. Tel.: +39.329.2112367 - Fax: +39.049 .8215672 . E-mail: patrizia.pavei@sanita.padova.it

Key words: recurrent varices, ultrasound foam guided sclerotherapy.

Received for publication: 4 August 2014.

Revision received: 8 October 2014.

Accepted for publication: 10 October 2014.

This work is licensed under a Creative Commons Attribution 3.0 License (by-nc 3.0).

(C) Copyright P. Pavei et al., 2014

Licensee PAGEPress, Italy

Veins and Lymphatics 2014; 3:4655

doi:10.4081/vl.2014.4655

and Tessari method was used to form the sclerosant foam (SF). ${ }^{12}$

$\mathrm{C}$ of the CEAP classification of the inguinal recurrences was as follows: $89 \mathrm{C} 2,37 \mathrm{C} 3,19$ C4, 8 C5 and $2 \mathrm{C} 6$. As for popliteal recurrences we had $17 \mathrm{C} 2,7 \mathrm{C} 3,2 \mathrm{C} 4,1 \mathrm{C} 5$ and $1 \mathrm{C} 6$ (Table 2 ). Some of the cohort data are summarized in Table 3.

Patients with neovascularization were treated with either polidocanol (POL) (Atossisclerol, Kreussler Pharma, Wiesbaden, Germany) or sodium tetradecylsulphate (STS) (Fibrovein, STD Pharmaceutical Ltd., Hereford, UK) in low concentration and low doses. More in detail, $0.3-0.5 \%$ POL or STS SF was injected under ultrasound guidance for vein having diameters $<3 \mathrm{~mm}$ and $0.5-1 \%$ POL/STS sclerosant foam was used for higher vein diameters. CEAP (C) distribution of the 142 neovascularization cases was: 99 C2, 29 C3, 12 C4 and 2 C5. Patients underwent from 1 to 3 sessions [mean $2.2 \mathrm{~mm}$ and standard deviation (SD) 0.37], with 4 to $10 \mathrm{~mL}$ per session (mean 6.2 and SD 1.58).

The patients with residual SFJ or SPJ stump (155 inguinal recurrences and 28 popliteal ones) were treated with $1 \%$ STS SF in cases of residual SFJ/SPJ diameter up to $5 \mathrm{~mm}$, while for larger veins 3\% STS was used. Patients underwent 1 to 3 sessions (mean 2,1 and SD 0.66 ) with 4 to $10 \mathrm{~mL}$ of foam injected per session (mean 7.8 and SD 1.42).The sessions were performed once a week for three consecutive weeks. After each session a short-stretch elastic bandage was applied for $24 \mathrm{~h}$ and then patients wore a II class elastic stocking for other 20 days. No additional sessions were allowed during the follow up period. Good clinical results were defined as the absence of clinical evident varices. Residual veins were recorded on drawings. 


\section{Results}

In the group of neovascularization the 3-5 years (mean 4 and SD 0.73) follow up revealed good results, defined as the absence of clinical evident varices and/or of any reflux in the injected site(s) at the CDU examination. More in detail 129 patients $(90.8 \%)$ had no clinical recurrence nor reflux at the groin neovascularization site and 13 patients (9.2\%) showed recurrent varicose veins.

As to the second group, characterized by the residual stump-based recurrence, a few differences were observed between inguinal and popliteal findings.

Among the 155 patients with inguinal recurrence, 4 were lost in the follow up, 1 for personal problems, 1 for health problems and 2 were no longer contactable (Figure 1). At 1 year follow up (151 patients) CDU showed complete occlusion of the treated veins in 132 patients (87\%) and no clinical recurrence was highlighted; at 2 years 98 patients completed the follow up: 83 patients (85.7\%) had good clinical results, while CDU showed complete occlusion in 76 patients (77.5\%). Finally at 3-5 years (mean 4.4 and SD 0.77), 75 patients completed the follow up and the CDU examination showed a $72 \%$ occlusion rate (54 patients), while the clinical recurrence was $20 \%$ (15 patients).

The 28 popliteal recurrences had a $3-5$ years (mean 4.3 and SD 0.76 ) follow up with $60.7 \%$ of complete occlusion at CDU (17 patients) and absence of varices. The analysis of patients where CDU highlighted recanalization of the treated stump and network ( $40 \%$ of the total), showed that $13 \%$ of patients had recanalization without visible varices and $27 \%$ of them showed recanalization with clinical evident varicose veins. The clinical recurrent varices were smaller than the pre-treatment ones in the vast majority of the cases.

As for complication we observed: i) gastrocnemial vein thrombosis in $0.2 \%$ of the cases; ii) minor neurological problems, namely visual disturbances and migraine, in $0.1 \%$; iii) $2.8 \%$ incidence of superficial vein thrombosis; iv) pigmentation in $3.9 \%$ of the patients; and v) light to mild post-treatment pain in $16.5 \%$ of the cases (Table 4).

\section{Discussion}

We divided our patients with recurrent varicose veins into 2 groups: group A with neovascularization at the groin area, and group B with new large varices emanating from a residual stump of the previously operated junction. In fact literature data show that smaller veins have better results with sclerotherapy

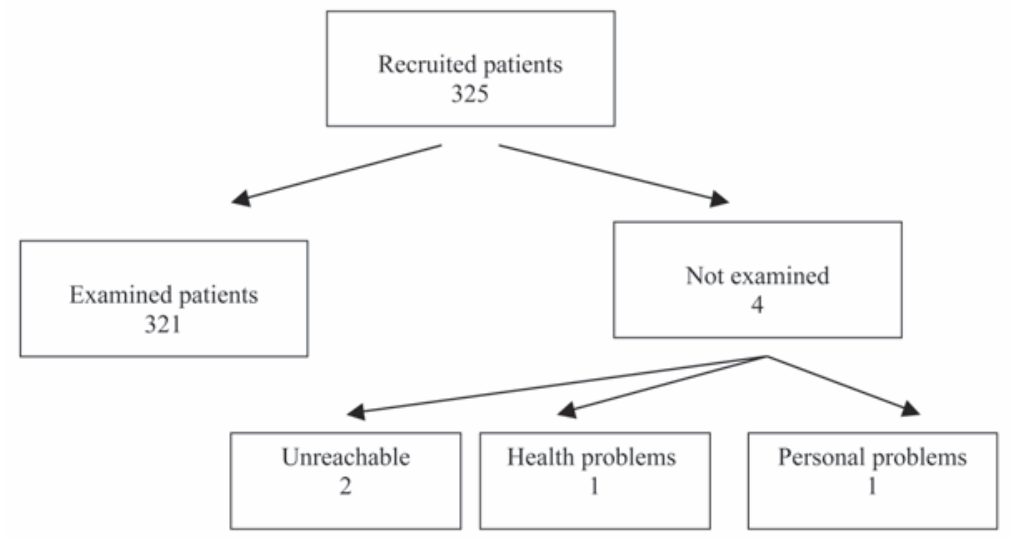

Figure 1. Recruited patients.

Table 1. Type of recurrences.

\begin{tabular}{llc} 
& Kind of recurrence & Patients \\
$\mathrm{C} 0, \mathrm{C} 1$ & - & 0 \\
$>=\mathrm{C} 2$ & Same site & 142 \\
& Neovascularization & 155 \\
& Inguinal residual stump and varicose network & 28 \\
& Popliteal residual stump and varicose network & 325 \\
\hline Total & &
\end{tabular}

Table 2. Clinical-Etiology-Anatomy-Pathophysiology classification (CEAP) classification of the treated patients.

\begin{tabular}{lcc} 
CEAP class & Patients & $\%$ \\
$\mathrm{C}_{0}$ & 0 & 0 \\
$\mathrm{C}_{1}$ & 0 & 0 \\
$\mathrm{C}_{2} \mathrm{E}_{\mathrm{p}} \mathrm{A}_{\mathrm{s}} \mathrm{R}$ & $89+17+99$ & - \\
$\mathrm{C}_{3} \mathrm{E}_{\mathrm{p}} \mathrm{A}_{8} \mathrm{R}$ & $37+7+29$ & - \\
\hline $\mathrm{C}_{4} \mathrm{E}_{\mathrm{p}} \mathrm{A}_{\mathrm{s}} \mathrm{R}$ & $19+2+14$ & - \\
$\mathrm{C}_{5} \mathrm{E}_{\mathrm{p}} \mathrm{A}_{\mathrm{s}} \mathrm{R}$ & 8 & - \\
$\mathrm{C}_{5} \mathrm{E}_{\mathrm{p}} \mathrm{As}+{ }_{\mathrm{p}} \mathrm{R}$ & $2+1$ & - \\
$\mathrm{C}_{6} \mathrm{E}_{\mathrm{p}} \mathrm{A}_{\mathrm{sp}} \mathrm{R}$ & 3 & - \\
\hline
\end{tabular}

Table 3. Cohort data.

\begin{tabular}{lcc} 
Patients' characteristics & Range & \\
Gender F/M & $232 / 93$ & $38-78$ \\
Mean age & 58.63 & $2.35-30.33$ \\
\hline Years from the first surgery (mean) & 11.44 &
\end{tabular}

Table 4. Complications after ultrasound guided foam sclerotherapy.

\begin{tabular}{lc} 
Complications & Patients (\%) \\
Gastrocnemius vein thrombosis & $0.2 \%$ \\
Superficial vein thrombosis & $2.8 \%$ \\
\hline Minor neurological complications & $0.1 \%$ \\
Post-treatment pain & $16.5 \%$ \\
\hline Pigmentation & $3.9 \%$
\end{tabular}

[Veins and Lymphatics 2014; 3:4655] 
than larger ones. ${ }^{13-15}$

In particular neovascularization ${ }^{16}$ is characterized by fragile, thin walled varices, hardly treatable with surgery and not eligible for endovascular treatments. ${ }^{7,8}$ On the contrary these features make these vessels well suitable for sclerotherapy.

Our 3-5 years follow-up results confirm this trend with an overall success of $90.8 \%$ in 142 patients with neovascularization (group A).

Conversely UGFS of larger varices emanating from the sapheno-femoral or sapheno-popliteal junction residual stump resulted in a higher recanalization rate at CDU examination and in a slightly higher clinical recurrence rate.

Literature data show a contrasting evidence as to the best option for treatment of recurrent varices. ${ }^{17-19}$

In 2008 we published a paper on redo surgery of the SFJ in 51 patients who were operated between 1996 and $2004 .{ }^{20}$ At 5 years follow up $31.4 \%$ of them had no clinical recurrence, $29.9 \%$ showed new varices; $5.8 \%$ residual varices. CDU investigation of these patients showed $23.5 \%$ neovascularization rate at the groin and $9.8 \%$ patients presented with new large varices coming from the re-operated junction, that is to say $33.3 \%$ had a true relapse after redo surgery at the groin. Therefore we decided to treat patients with a less invasive technique, namely with UGFS. According to our personal experience and data, recurrent varices may be treated with UGFS in the vast majority of the cases and efficacy and safety of this method has been demonstrated in our and other authors' clinical series. ${ }^{9-10}$

Recurrence related to neovascularization at the groin or at the popliteal area respond well to UGFS, whereas recurrent varices fed by a residual stump of the SFJ or SPJ shows slightly worse outcomes, especially for SPJ-based recurrence

Probably higher concentrations and higher volumes of sclerosant foam may be of help to improve UGFS furthermore in this second group of patients.

A strict CDU-based follow up and, if necessary, an early re-treatment should be considered in this second group of patients with residual stump-based recurrence, especially at popliteal fossa.

\section{Conclusions}

In conclusion, our data show that UGFS is the treatment of choice in case of neovascular- ization-based varicose recurrence. Similarly in cases of recurrence emanating from the residual stump of the operated junction UGFS may be the first choice treatment. Finally in our recent practice we have included phlebectomy of the large varices along the limb, in order to decrease the total volume of injected sclerosant foam and to speed-up the treatment results.

UGFS in our patients showed good mid-term results, and furthermore it proved to be a well tolerated technique, largely preferred by previously operated patients, extremely safe, costeffective and easily repeatable.

\section{References}

1. Perrin MR, Guex JJ, Ruckley CV, et al. Recurrent varices after surgery (REVAS), a consensus document. Cardiovasc Surg 2000;8:233-45.

2. Perrin MR, Labropoulos N, Leon LR. Presentation of the patient with recurrent varices after surgery (REVAS). J Vasc Surg 2006;43:327-34; discussion 334.

3. Perrin M, Allaert FA. Intra- and interobserver reproducibility of the recurrent varicose veins after surgery (REVAS) classification. Eur J Vasc Endovasc Surg 2006;32:326-2.

4. Van Rji AM, Jones GT, Hill GB, et al. Neovascularization and the recurrent varicose veins: more histologic and ultrasound evidence. J Vasc Surg 2004;40:296-302.

5. Jones L, Braithwaite BD, Selwyn D, et al. Neovascularization is the principal cause of varicose vein recurrence: results of a randomized trial of stripping the long saphenous vein. Eur J Vasc Endovasc Surg 1996;12:442-5.

6. El Wajeh Y, Giannoukas AD, Gulliford CJ, et al. Saphenofemoral venous channels associated with recurrent varicose veins are not neovascular. Eur J Vasc Endovasc Surg 2004;28:509-94.

7. Royle JP. Recurrent varicose veins. World J Surg 1986;10:944-53.

8. Labropoulos N, Touloupakis E, Giannoukas $\mathrm{AD}$, et al. Recurrente varicose veins: investigation of the pattern and extent of reflux with color flow duplex scanning. Surgery 1996;119:406-9.

9. Darvall KAL, Batev GR, Adam DJ, et al. Duplex ultrasound outcomes following ultrasound guided foam sclerotherapy of symptomatic recurrent saphenous varicose veins. Eur J Vasc Endovasc Surg 2011;42:107-14

10. Kakkos ST, Bountouroglou DG, Azzam M, et al. Effectiveness and safety of ultrasound guided foam sclerotherapy for recurrent varicose veins: immediate results. J Endovasc Ther 2006;13:357-74.

11. Nicolaides AN. Investigation of chronic venous insufficiency. A consensus statement. Circulation 2000;14:1-38.

12. Breu FX, Guggenbichler S, Wollmann JC. 2nd European Consensus Meeting on Foam Sclerotherapy, 28-30 April 2006 Tegernsee, Germany. VASA 2008;37:1-32.

13. Myers KA, Jolley D, Clough A, Kirwan J. Outcome of ultrasound guided sclerotherapy for varicose veins: medium term results assessed by ultrasound surveillance. Eur J Vasc Endovasc Surg 2007;33: 116-21.

14. Coleridge-Smith P. Chronic venous disease treated by ultrasound guided foam sclerotherapy. Eur J Vasc Endovasc Surg 2006;32:577-83.

15. Cavezzi A, Frullini A, Ricci S, Tessari L. Treatment of varicose veins by foam sclerotherapy: two clinical series. Phlebology 2002;17:13-8.

16. Turton EPL, Scott DJA, Richards SP, et al. Duplex-derived evidence of reflux after varicose vein surgery: neoreflux or neovascularisation? Eur J Vasc Endovasc Surg 1999;17:230-3.

17. Société Française de Phlébologie. Les récidives variqueuses après chirurgie. Phlébologie 1998;51:387-430.

18. Bradbury AW, Stonebridbge PA, Callam MJ, et al. Recurrent varicose veins: assessment of the saphenofemoral junction. Br J Surg 1994;81:373-5.

19. De Maeseneer MG, Vandenbroeck CP, Hendriks JM, et al. Accuracy of duplex evaluation one year after varicose vein surgery to predict recurrence at the sapheno-femoral junction after five years. Eur J Vasc Endovasc Surg 2005;29:308-12.

20. Pavei P, Vecchiato M, Spreafico G, et al. Natural history of recurrent veins undergoing reintervention: a retrospective study. Dermatol Surg 2008;34:1672-82. 